\title{
Andreas Wiebel
}

\section{Ein Elefant auf Abwegen \\ Indiens fundamentaler Politikwechsel unter Narendra Modi}

\section{Abschied vom Nehruvianismus}

Make in India hieß die Initiative, mit der Narendra Modi im September 2014 seine Arbeit als 15. Premierminister Indiens aufnahm. In nahezu allen Politikbereichen wurden damit die Schleusen für (inter-)nationales Kapital geöffnet, um das zu erreichen, was die (Finanz-)Welt seit über einem Jahrzehnt von Indien erwartet und Modi seinen WählerInnen versprochen hatte: Wachstum, Wachstum, Wachstum. Nicht jedoch die Frage, ob und wie dieses Wachstum zustande kommt, sondern der Zusammenhang zwischen technologisch-ökonomischer Expansion und religiösem Fundamentalismus soll Gegenstand der Betrachtung sein. Dieser Artikel ist der Versuch, die Gründe für den Wahlerfolg Narendra Modis und die Bedeutung des Hinduismus für die Durchsetzung einer neoliberalen Agenda zu benennen.

Narendra Modi ist bei aller Abgrenzung zur bisherigen politischen Elite Indiens kein unbeschriebenes Blatt, sondern gehört zum harten Kern der rechtsgerichteten Indischen Volkspartei (Bharatiya Janata Party, BJP). Als solcher vertritt er nicht nur abermillionen Hoffnungen der Mittelschicht nach einem Wirtschaftswunder, politischer Stabilität, moderner Infrastruktur und effizienter Regierungsarbeit, sondern auch die Ressentiments der gegen westliche Werte, Modernisierungsprozesse und religiöse Toleranz gerichteten HindutvaBewegung. Wie lassen sich die unterschiedlichen Interessen umsetzen und für welchen Wechsel hat der Premier sein Mandat erhalten?

Dass der politische Wechsel, der mit der absoluten Mehrheit der BJP im Parlament einhergeht, Auswirkungen auf die indische Gesellschaft hat, liegt auf der Hand. Deshalb kann bereits nach eineinhalb Jahren Regierungszeit eine erste Bilanz gezogen werden, die mit Blick auf die politische Kultur und den vom Pluralismus geprägten Charakter Indiens negativ ausfällt. Bereits kurz nach dem Amtsantritt Modis gab es Meldungen über Vandalismus in Kirchen, gefolgt von Ausschreitungen wegen des Konsums von Rindfleisch und kommunalistischen Aggressionen vor allem gegen Menschen muslimischen Glaubens, was ein Klima 
der Einschüchterung und Bedrohung in liberalen Kreisen zur Folge hatte und zuletzt in der Ermordung mehrerer linker AktivistInnen und OppositionspolitikerInnen gipfelte, die sich zum Beispiel gegen Aberglauben oder arrangierte Ehen engagierten. Mindestens ebenso besorgniserregend wie diese menschenverachtenden Geschehnisse sind die Veränderungen staatlicher Strukturen. So wurden nicht nur die - zugegebenermaßen obsolete - Planungskommission abgeschafft, sondern auch wichtige Posten nationaler Kultur- und Bildungseinrichtungen mit reaktionären BJP-HardlinerInnen besetzt, die im banalsten Fall nur die Kuss-Szenen aus James-Bond-Filmen zensieren.

Unterm Strich verhärtet sich der Verdacht, dass die tragenden Säulen des von Jawaharlal Nehru begründeten Staatsverständnisses, welches auf Säkularismus, Antiimperialismus, Pluralismus und Sozialismus fußte, zugunsten einer nationalidentitären Ideologie auf der Basis des Hinduismus (Hindutva) ausgetauscht werden sollen. ${ }^{I}$ Das wird, wo der staatliche Arm nicht hinreicht oder zivilisiert bleiben muss, von paramilitärischen Organisationen wie dem Rashtriya Sevak Sangh (kurz RSS, nationales Freiwilligenkorps) durchgeführt, die sich aufgrund des erdrutschartigen Wahlsiegs der BJP bemächtigt fühlen, den kulturellen Wandel herbeizuführen. Inwiefern Premierminister Modi über die Aktivitäten seiner Partei und GesinnungsgenossInnen informiert ist oder gar dafür verantwortlich gemacht werden kann, muss an dieser Stelle unbeantwortet bleiben. Generell müssen allgemeine Aussagen über Indien immer mit einem „Haltbarkeitsdatum“ (Trojanow 2007: 3) versehen werden, da in Indien wenig so konstant ist wie der Wandel. Darüber hinaus gilt es, das Verhältnis zwischen den Informationen, die mehr mit einer gesellschaftspolitischen Neuorientierung im Allgemeinen zu tun haben und jenen, die mehr die Person Modi im Besonderen betreffen, im Blickfeld zu behalten. Auch wenn sich diese Entwicklungen zutiefst beeinflussen, führt die Polarisierung auf die Figur Narendra Modi - wie es von westlichen Medien oft unternommen wird - zu einer verkürzten Sicht der Dinge.

\section{Narendra Modi - eine Projektionsfläche?}

Dass Indien ökonomische und soziale Entwicklung benötigt, daran besteht kein Zweifel. Die Defizite der maroden Infrastruktur (Elektrizität, Wasser, Verkehr), beim Umwelt-, Natur- und Klimaschutz, bei Antikorruptionsgesetzen, sanitären Anlagen oder staatlichen Bildungseinrichtungen sind hinlänglich bekannt. Dazu

1 Vgl. Präambel der Republik Indien, seit 1976: „We, the people of India, having solemnly resolved to constitute India into a sovereign socialist secular democratic republik and to secure to all its citizens." 
kommt die seit Jahrzehnten ungelöste Armuts- und Hungerproblematik ${ }^{2}$ sowie das Defizit an Millionen neuen Arbeitsplätzen, da die indische Bevölkerung jedes Jahr um die Größe der australischen wächst (vgl. Tharoor 2005: 274). Eine herausfordernde Aufgabe für jeden Politiker, gleich welcher Couleur. Deshalb hat Modi es sich zur Aufgabe gemacht, den großen Wandel einzuläuten. Er, der Macher, der bereits das Bundesland Gujarat auf Vordermann gebracht hatte, versprach das Gleiche für Gesamtindien. ${ }^{3}$ Wie ein „Evangelist“ (Prasannaranjan 2014: 14) sprach er von besseren Tagen, die kommen würden und von mehr Reichtum für alle, der durch mehr Wirtschaftswachstum erreicht werden könne - ganz im Unterschied zu Rahul Gandhi, dem Kandidaten der Kongresspartei, der sich an ausgetrocknete Sozialprogramme seiner politischen Vorfahren klammern müsse, die nur noch den Stillstand zu verwalten vermochten. Immer wieder erklärte Modi das Kongressprogramm denn auch als Mythos: Die Situation der Armen könne nicht mit Umverteilung verbessert werden, sondern nur durch Modernisierung. Im Glauben ans 21. Jahrhundert gab Modi die Parole aus: „Third Worldism is dead for us!" (Prasannaranjan 2014: 17) In der Praxis bedeute das: 100 Smart Cities, mehr Indian Institutes of Technology (IIT), Indian Institutes of Management Shillong (IIMS) und All India Institutes of Medical Sciences (AIIMS), Hochgeschwindigkeitszüge, Berufsförderungsprogramme, Straßen, Urbanisierung, Industrialisierung u.v.m. Indien soll während seiner Amtszeit auf chinesisches Niveau gebracht werden.

Nachdem die Sehnsüchte der modernen indischen Massen längst globalisiert und viele junge InderInnen in ihren täglichen Realitäten vom Gedanken des materiellen Reichtums und von der Vorstellung erfüllt sind, dass sie ein besseres Leben haben werden als ihre Eltern (vgl. Khilnani 2014: 16), hatte ein Politiker, der unentwegt Wechsel, Wachstum und Wohlstand versprach, leichtes Spiel. Hinzu kam Modis neuer Führungs- und Kommunikationsstil. Er hat keine Berührungsängste mit moderner Telekommunikation. Im Gegenteil: Zur Verbreitung seiner politischen Agenda und für den Kontakt zu BürgerInnen nutzt Modi soziale Medien so intensiv wie kaum ein anderer Politiker auf der Welt. 40 Millionen Menschen folgen ihm aktuell auf Facebook und Twitter. Seit Juni 2015 hat er überdies seine eigene App (für Smartphones oder Tabletcomputer) und ein Team von zwanzig MitarbeiterInnen ist rund um die Uhr mit Updating beschäftigt (vgl. Tilman 2015). Die Affinität zu moderner Technologie zeigte sich auch in Modis aufwendiger Wahlkampagne nach US-Vorbild. Von Uwe Maass,

2 Indien führt mit 300 Millionen Menschen nach wie vor die Armutsstatistik der Vereinten Nationen an.

3 Von 2001 bis 2014 war Modi Chief Minister (Ministerpräsident) von Gujarat und schob das Land beim Wirtschaftswachstum auf Platz eins im nationalen Vergleich. 
einem gebürtigen Rheinländer und Fachmann für 3D-Technologie, ließ Modi sich bei 1.680 Wahlkampfauftritten als Hologramm auf die Bühne projizieren, wodurch er einen kaum zu überschätzenden Vorteil gegenüber anderen KandidatInnen und immensen Einfluss auf die ZuhörerInnen erzielte. Der ModiAvatar war im hochgradig personalisierten Wahlkampf quasi omnipräsent und der Unterschied zwischen „Realität und Täuschung für die Augen von Millionen von potenziellen Wählern kaum mehr wahrnehmbar. (...) Die meisten Zuschauer haben nicht verstanden, dass da nicht der echte Modi auf der Bühne stand und zu ihnen sprach " (Skischally 2014: 115), sondern ein Hologramm. Dieses sprach von einem anderen Indien, das mit ihm erreicht werden könne, und davon, dass speziell die Armen das Rückgrat des indischen Wachstums ausmachten.

\subsection{Modis Image beginnt, zu bröckeln}

Am 16. Mai 2014 bestätigte sich, dass Narendra Modi die Wahl zum 14. Ministerpräsidenten Indiens gewonnen hatte. Noch nie zuvor konnte eine hindunationalistische Partei die absolute Mehrheit in Indiens nationalem Parlament erringen. ${ }^{4}$ Von großen Erwartungen und mehrheitlich positiv gestimmter Berichterstattung begleitet, nahm Modi die Regierungsgeschäfte auf, um den großen Kurswechsel einzuläuten: Digitalisierung und Beschleunigung bürokratischer Prozesse durch Zentralisierung der Entscheidungsgewalt im Büro des Premiers, Senkung der Inflationsrate, Reduzierung der Umsatzsteuer, Erhöhung ausländischer Direktinvestitionen, Privatisierung von Bergbaurechten, Eröffnung von 125 Millionen Bankkonten für mittellose Familien, Einwerbung von Investoren zum Ausbau der Solarstromkapazität, Proklamation des Welt-Yoga-Tages. Zudem verpflichtete Modi via Online-Login BeamtInnen zur Anwesenheit am Arbeitsplatz und zur Entrümpelung der Regierungsbehörden. Auf den ersten Blick kann sich diese Bilanz sehen lassen. Allerdings stellten vieles davon bisher vor allem medienwirksame Kampagnen dar, wie die Initiative Make in India zur Schaffung neuer Industriestandorte oder Swachh Bharat zur Sensibilisierung für die Verschmutzung indischer Städte, deren Erfolge man erst in einigen Jahren beurteilen können wird. ${ }^{5}$

4 Und das, obwohl die BJP nur 40 Prozent der Stimmen erhalten hat, was mit dem indischen Mehrheitswahlrecht zusammenhängt. Zieht man überdies die Zahl der NichtwählerInnen ab, bleiben netto nicht mehr als 20 Prozent für den Wahlsieger übrig.

5 Die Kampagnen sind ein Anschauungsbeispiel für Modis Art, Politik zu machen. Wie bereits im Wahlkampf setzt der indische Premierminister auf eine starke persönliche Präsenz und auf die Kraft der neuen Medien. Am Tag der Veröffentlichung der Sauberkeitin-Indien-Kampagne (Swachh Bharat) griffer medienwirksam selbst zum Besen und fegte 
Weil Modi alles an das scheinbar ewige Gesetz des Wachstums gekoppelt hat, musste er den Kernteil seiner Wahlversprechen - mehr Jobs, mehr Fortschritt - bisher schuldig bleiben. Insbesondere vonseiten der ungeduldiger werdenden Corporate World, die einen "großen Knall der Liberalisierung“ (Harneit-Sievers 2015) erwartet hatte, wird beklagt, dass tiefe strukturelle Reformen wie die weitere Privatisierung von Staatsbetrieben, die Angleichung der Mehrwertsteuer, die Erleichterung von Firmengründungen oder die Bereitstellung von Land für Industrie- und Infrastrukturprojekte bisher ausgeblieben sind (vgl. Barry 2015). Einerseits hat dies mit dem stark verankerten Föderalismus Indiens zu tun, das heißt mit der Mehrheit oppositioneller Parteien in der zweiten Kammer (Oberhaus, Rajya Sabha) sowie einer veralteten, zersplitterten Bürokratie; zum Großteil jedoch mit dem Überentwurf von Modis Image als fleißiger Administrator, durchsetzungsstarker Manager, Entwicklungsmann und charismatische Führungspersönlichkeit. Die Realität hat sich dem überlebensgroßen Image nicht angepasst, das er während des Wahlkampfs mit Hilfe von PR-Agenturen von sich geschaffen hat. Und weil sein Image des Wechsels einem Superhelden glich und damit größer als er selbst war (vgl. Barry 2015), wird die Einlösung seiner Versprechungen nicht aufgehen, selbst wenn er für eine zweite Amtszeit wiedergewählt wird, wovon derzeit auszugehen ist. Obwohl Modis Kompetenzen langsam als wohlklingende Rhetorik und große Show erkannt werden, ist seine Popularität kaum gesunken (ebd.). Der Großteil der Leute, die ihn gewählt haben, ist verständnisvoll und sich darüber im Klaren, dass Indiens Probleme nicht im Handumdrehen gelöst werden können.

Eine andere Opposition gegen Modi bzw. die BJP entstand jüngst auf Seiten der Subalternen (Scheduled Tribes, Scheduled Castes, Other Backward Classes), für die die Modi-Politik bisher keine positiven Auswirkungen gebracht hat. Im Gegensatz dazu hatte die Kongresspartei, auch aufgrund des Drucks der Linken, noch leichte Anstrengungen zum Aufbau eines rudimentären Sozialstaates unternommen (Recht auf Arbeit, Recht auf Information, Recht auf Bildung, Recht aufErnährung). Doch nun wurden diese Programme durch die Modi-Regierung mit Verzögerungsstrategien oder mangelhafter Finanzierung größtenteils wieder kassiert (vgl. Purkayastha 2015). Während ca. 200 Millionen InderInnen der Mittelschicht vom indischen Entwicklungsmodell profitieren, bleibt der Großteil der arbeitenden Bevölkerung, welcher in Indien immer noch in der Landwirtschaft beschäftigt ist, von den Früchten der kapitalistischen Expansion ausgeschlossen (vgl. Amir 2007: 710). Nebst der Wahl in Delhi (Februar 2015) kann vor allem die bedeutende State Election in Bihar (Oktober 2015), in der die BJP nur einen

den Vorplatz eines Polizeigebäudes in Delhi. Dabei rief er berühmte Cricketspieler und Filmstars auf, es ihm gleichzutun. 
Bruchteil der angestrebten Mandate erringen konnte, in Zusammenhang mit ihrer bauernfeindlichen Politik gebracht werden. Vorausgegangen war der Streit über die geplante Reform des Gesetzes zum Grunderwerb (Land Acquisition Act), die den Landkauf, das heißt die Landenteignung für Industrieunternehmen, erleichtern sollte und die Kleinbauern ohne Kompensation zurückließ, weshalb letztere sich mit der Opposition zusammenschlossen. Westliche Nationen konnten ihren Aufstieg noch auf Kosten ihrer Kolonien durchführen, Indien kolonisiert sich demgegenüber gerade selbst (vgl. Roy 2014).

\subsection{Nerviges Establishment}

Indiens Politik ist geprägt von Politikerdynastien, wovon die Nehru-GandhiFamilie sicherlich die namhafteste darstellt und bisher dreimal den Regierungschef stellte. Auch auflandespolitischer und kommunaler Ebene werden Mandate häufig im erweiterten Familienkreis vererbt. Modi hingegen erscheint als anderer Politikertyp, jedenfalls nicht als Berufspolitiker aus der intellectual urban middle class, sondern als Aufsteiger. Die Tatsache, dass er aus einer niederen sozialen Kaste stammt, wurde im Wahlkampf gezielt eingesetzt und spielte als Identifikation für viele InderInnen aus ähnlichen Verhältnissen sicherlich eine große Rolle. Modi weiß, was Macht bedeutet, er hat sich vom Teeverkäufer über die Arbeit als RSS-Agitator zum Premierminister hochgearbeitet und scheut nicht davor zurück, seine Macht einzusetzen.

Der Absturz der Kongress-Partei, die damit zumindest vordergründig verbundene Abkehr von sozialdemokratischen Ideen von Jawaharlal Nehru, hatte ein Machtvakuum zur Folge, in das die BJP vorgerückt ist. Wie bereits 1998, als mit Atal Behari Vajpayee der letzte BJP-Politiker an die Macht gekommen war, hatte die Kongresspartei auch dieses Mal im Vorfeld der Wahl große Fehler gemacht. ${ }^{6}$ So gab es Korruptionsskandale um Mobilfunklizenzen (2G Scam), Kohleabbaurechte (Coalgate), die Etihad-Jet-Airlines-Beteiligung oder die Amnestie für verurteilte Abgeordnete; ganz zu schweigen von der Offensive gegen die Naxaliten (Operation Green Hunt), die von der Modi-Regierung inzwischen mit noch mehr Militär weitergeführt wird.

Was den politischen Hinduismus (Hindutva) lange Zeit zurückgehalten hatte, war die Präsenz einer säkularen Alternative, die eine vernünftigere Antwort auf

6 Bei einer detaillierteren Analyse müsste allerdings benannt werden, dass die strukturellen Probleme tiefer liegen. Selbst das ehrlich ausgeführte sozialdemokratische Politikmodell der Kongresspartei wäre über kurz oder lang im Kontext globalisierter Märkte und Bedürfnisse an seine Grenzen gestoßen. Indien muss erkennen, dass es seine Probleme nicht durch das Wachstums-Rezept des Westens gelöst bekommt. 
die sozialen und ökonomischen Fragen der Gegenwart gegeben hat als heiliger Boden (rashtra), Abstammung (jati) und Kultur (sanskriti) (vgl. Gupta 2011: 165). Der gegenwärtige Hindunationalismus ,präsentiert sich selbst als indigene Alternative zum nehruvianischen Nations- und Entwicklungsmodell der Kongresspartei, deren Säkularismus und Universalismus als verwestlichte Fortsetzung kolonialer Herrschaft kritisiert wird“" (Framke/Tschurenev 2010: 73). Nach der 1991 vollzogenen Liberalisierung der indischen Wirtschaft durch den späteren Ministerpräsidenten Manmohan Singh stand Indien zwar als Hoffnungsträger des internationalen Kapitals 15 Jahre überbewertet auf westlichen Anlageplänen (vgl. Roy 2014), hatte aber innenpolitisch wenig eigene Konzepte zu bieten, um den oben skizzierten Herausforderungen zu begegnen. 1995 kam der Beitritt zur WTO, der die Privatisierung des Wassers in Indiens Großstädten zur Folge hatte, und 2001 die Öffnung der Märkte für Agrarimporte (Einsatz internationaler Düngemittel, genetisch veränderten Saatguts, Reduzierung von Agrarsubventionen), was die Situation der Bauern und Bäuerinnen vollends verschlechterte. Inzwischen ist die Zahl der Suizide unter ihnen auf über 300.000 gestiegen.

Die Wahl Modis kann deshalb vor allem als Abwahl der Kongresspartei verstanden werden, die über drei Viertel ihrer Mandate verlor. Im Umkehrschluss ist dies die halbe Antwort auf die Frage, wie die BJP so erdrutschartig an die Macht kommen konnte, nämlich resultierend aus der schlechten Leistung der Kongresspartei, was den Modi-Effekt gewaltig relativiert. Nichtsdestotrotz hat Modis Wahlerfolg auch etwas mit der Sehnsucht eines Teils der indischen Gesellschaft nach einem starken Anführer zu tun, der eine neue, selbstbewusste Identität, ein neues Narrativ verkörpert.

\section{Hindutva oder die Safranisierung des Subkontinents}

Man muss sich vergegenwärtigen, dass Indien ein in weiten Teilen nach wie vor zutiefst spirituelles Land ist. Im Unterschied zu den meisten anderen Ländern der Welt gibt es in Indien nach dortigem Zeitempfinden eine seit 5.000 Jahren ungebrochene Tradition von Philosophie und Religion. „Der Besuch bedeutender Tempel und Pilgerorte, regelmäßiges rituelles Fasten und die Hinwendung zu tradierten, religiösen Praktiken oder zu einem persönlichen Guru (Lehrer) haben mit der Globalisierung und ihren weltlichen Verlockungen nicht etwa abgenommen, sondern - insbesondere in der städtischen Mittelschicht - seit den 80er Jahren eher noch an Zulauf gewonnen" (Kakar 2011: 133). Allerdings ist der Hinduismus als einheitliche Religion ein europäisches Missverständnis, denn im Gegensatz zu anderen Religionen kennt der Hinduismus keine allgemein verbindliche Glaubenslehre, oder anders formuliert: „Im Hinduismus findet 
sich für nahezu alles auch sein Gegenteil“" (Hörig 2007: 4). Die drei wichtigsten religiösen Bewegungen heute sind der Vishnuismus, Shivaismus und Tantrismus (vgl. Ceming 2004: 101), welche sich in der Praxis stark unterscheiden. Ebenso ist auch der Name Indien eine westliche Fremdbezeichnung. Die Briten leiteten das Wort schlichtweg von der geografischen Umgebung ab: für die Region, die sich hinter dem Fluss Indus befindet. Sprechen InderInnen über ihr Land, so benutzen sie entweder das Wort Bharata, welches auf einen mythischen König aus dem Rigveda rekurriert und eine religiöse Konnotation besitzt, oder das Wort Hindustan - als Äquivalent zu Pakistan -, welches aus dem Persischen kommt und eigentlich mehr die geografische Verfasstheit betont, aber auch unter nationalistisch gesinnten SprecherInnen Verwendung findet. Da die Auseinandersetzung zwischen Indien und Bharat bzw. Hindustan andauert, ließe sich aus europäischer Begriffsperspektive sagen, dass Indien postmodern ist, ohne jemals modern gewesen zu sein, denn dadurch hat es immer viele Narrative nebeneinander gegeben: Die Veden, die Arier, die Moguls, die Kolonialen, die Unabhängigkeitsbewegung, die IT-Spezialisten etc. Nach den Gründern der modernen Republik Indien Mahatma Gandhi, Jawaharlal Nehru u.a. - sollte die religiöse Ausrichtung des Einzelnen beim Nationalgefühl keine große Rolle spielen, sondern eine private Angelegenheit bleiben: deshalb auch der Begriff Indien und nicht Bharat (vgl. Ceming 2004: 100). Per Verfassung kennt Indien keine Staatsreligion, im Gegenzug werden alle Glaubensbekenntnisse und religiösen Praktiken unterschiedslos respektiert und anerkannt. Diese Offenheit spiegelt sich in der offiziellen Flagge Indiens wider, deren Farben unter anderem die drei religiösen Grundströmungen Indiens repräsentieren: safrangelb für Hinduismus, grün für Islam und weiß für alle Minderheitenreligionen. Nehrus Traum war es, dass die Unabhängigkeitsbewegung über alle Landes-, Sprachen-, Kultur- und Religionsgrenzen hinweg InderInnen mit einer nationalen Identität schaffen würde. Im Unterschied zu den meisten modernen Nationen, die „das Ergebnis einer jahrhundertelangen Verschmelzung von Bevölkerungsgruppen bis zu dem Punkt [sind], wo die Elemente nicht mehr voneinander zu unterscheiden sind“" (Tharoor 2005: 177), bleiben im Falle Indiens mehr Differenzen als Gemeinsamkeiten übrig, da ein Bengali und ein Punjabi weder Ethnie noch Sprache oder Religion gemeinsam haben, mit denen sich eine nationale Identität bestimmen ließe. Was bleibt, ist die „Gemeinsamkeit der großen Differenzen“ (Tharoor 2005: 180).

\subsection{Hindu-Nationalismus statt Indien-Nationalismus}

Der skizzierte, im besten Sinne postmoderne Ansatz, wird jetzt durch ein Mononarrativ ersetzt: Bharat bzw. Hindustan soll das Land der Hindus sein. Also sind alle InderInnen, die in Indien leben, nach dem Verständnis der 
Hindu-Nationalisten Hindus oder müssen es werden. Die Geschichte scheint sich zu wiederholen. Wie auch in den 1990er Jahren erlebt der Hindu-Nationalismus, der auch als Hindu-Fundamentalismus bezeichnet werden kann, derzeit einen großen Aufschwung, was sich außer mit den Fehlern der Kongresspartei vor allem mit der radikalen wirtschaftlichen Liberalisierung, bei gleichzeitiger Säkularisierung und Integration Indiens in die globale Weltwirtschaft erklären lässt, begleitet von sozialen Spannungen und Verelendungserscheinungen. Das heißt, die wachsende wirtschaftliche Ungerechtigkeit führt zu Empörung bei den Armen und Benachteiligten, die wiederum von den Hindu-NationalistInnen ausgenutzt und in religiöse, kommunalistische Intoleranz kanalisiert wird.

Seine Ursprünge hat der Hindu-Nationalismus Ende des 19., Anfang des 20. Jahrhunderts als Widerstandsbewegung gegen den Kolonialismus und die "Dominanz europäischer Kultur und christlicher missionarischer Offensiven“ (Kakar 2011: 134). In den 1920er Jahren entstand mit der Gründung des RSS eine chauvinistische, radikal-hinduistische, paramilitärische Massenbewegung mit inzwischen sechs Millionen Mitgliedern und über 50.000 Ortsgruppen, in bewusster Abgrenzung zu Gandhis Politik der Gewaltlosigkeit, die starken Einfluss auf die Neuformulierung des Hinduismus hatte. ${ }^{7}$ Das Ziel des RSS war und ist es, „die in Kasten, Sekten und Lokaltraditionen zersplitterte HinduGemeinschaft zu einer Einheit zusammenzuschweißen“ (Kakar 2011: 135). Der Hindu-Nationalismus zieht seine Kraft dafür aus der „Tendenz zur Homogenisierung von Mythen und Symbolen der indischen Göttervielfalt" (Kakar 2011: 135). So ist insbesondere auf den Rama-Kult mit seiner Verehrung Ramas als königlicher Inkarnation des Gottes Vishnu hinzuweisen, mit dem reaktionäre Kulturinhalte legitimiert und durchgesetzt werden (vgl. Schiessl 2013: 25). ${ }^{8}$

Interessanterweise spielen bei der Verbreitung reaktionärer, nationalistischer Ziele moderne Technologien und Massenmedien eine große Rolle, die als Forum der Interessensdurchsetzung stark genutzt werden. Aber auch mythologische Fernsehserien erreichen höchste Einschaltquoten (vgl. Kakar 2011: 135). „Der Fundamentalismus ist eine Protest- und Widerstandsbewegung gegen den Li-

7 Der Neohinduismus (Ende 19. Jahrhundert) wird vor allem mit dem Namen Swami Vivekanandas in Verbindung gebracht. Er proklamierte einen Hinduismus der Toleranz und universalen Akzeptanz, welcher alle Religionen als wahr anerkennt und als Glaube nach dem Prinzip der Einheit in der Vielfalt funktionieren sollte. „Tatsächlich ist das die Art von Hinduismus, der von der großen Mehrheit der indischen Hindus praktiziert wird." (Tharoor 2005: 185f.)

8 Das Leben Ramas wird als ideales Menschsein stilisiert. Zum Beispiel die Hingabe, Unterwürfigkeit und Enthaltsamkeit seiner Frau Sita und ihre Reinigung durchs Feuer. Durch die Popularisierung der Epen Ramayana und Mahabharata werden Witwenverbrennung (Sati) oder rituelle Kastenvorschriften am Leben gehalten. 
beralismus, die Aufklärung und den Säkularismus. Aber er steht modernen Errungenschaften, insbesondere der Technik nicht feindlich gegenüber, sondern benutzt deren Möglichkeiten ausgiebig für seinen Kampf.“ (Ceming 2004: 104)

Die Basis hindunationalistischer Ideologie ist der Glaube, dass - wenn alle InderInnen ihre ursprüngliche Hindu-Identität anerkennten -, dies zur Einheit des Landes führen und die beste Verteidigung gegen alle Eindringlinge und separatistischen Bewegungen darstellen würde. „Der Begriff bezeichnet die Vorstellung, die Zugehörigkeit zur hinduistischen Religion habe Vorrang, weil sie die 'eigentliche Identität' der Völker des Landes ausmache." (Amin 2007: 712).9 Zwar dürften MuslimInnen oder ChristInnen ihren Glauben im Privaten weiter praktizieren, aber in der Öffentlichkeit wären sie angehalten, Loyalität gegenüber Hindu-Symbolen und Praktiken zu zeigen. „Deswegen behaupten auch die Fundamentalisten des RSS, dass sie, trotz ihrer anderslautenden Propaganda, nichts gegen Muslime und Christen in Indien haben, so sie sich denn hinduisieren." (Ceming 2004: 114) Es geht also primär nicht um religiöse Vorgaben, sondern um einen weitreichenden kulturellen Einfluss, das heißt, um einen cultural turn nach dem Motto: Hindi, Hindu, Hindustan.

Das politische Konzept für diese faschistoide Allianz aus Sprache, Religion und Region heißt Hindutva. Ein Begriff, der auf Vinayak Damodar Savarkar, einen der ideologischen Väter des Hindu-Nationalismus, zurückgeht. Wie Keshav Baliram Hegdewar, dem Gründer des RSS, ließ sich Savarkar von den Schriften der deutschen Nationalsozialisten inspirieren. Was für Adolf Hitler die Juden und Jüdinnen waren, sah Savarkar in den MuslimInnen (vgl. Schiessl 2013: 17). Hindutva ist also der Traum von der politischen Hindu-Einheit aller in Indien geborenen Menschen, wobei die Überhöhung der Hindu-Religionszugehörigkeit aus dem Universalitätsbegriff dieses Religionsverständnisses resultiert. „So betrachtet der Hindu-Nationalist den Hinduismus als universell, weil er auf einer ewigen, mystischen Wahrheit beruht“ (Kakar 2011: 140), deren Gesetzmäßigkeit, der Sanatana Dharma, die ewige Ordnung, das kosmische Gesetz für alle gelten soll (vgl. Ceming 2004: 102). Demnach sind alle anderen Religionen und Glaubenskonzepte nur eine Erscheinungsform des Hinduismus und letztlich alle Religionen hinduistisch.

9 „Die Verbundenheit und Loyalität Hindustans gegenüber findet für Hindunationalisten nicht nur durch die Verbundenheit zum Geburtsort statt, sondern auch auf spiritueller Ebene, [von der] Christen, Muslime und Juden von Hindunationalisten als strikt 'nichtindisch' ausgegrenzt werden. Die Argumentation ist, dass sie mit Indien immer nur über den 'Vaterland'-Punkt verbunden sein können, nie aber über den spirituellen, denn die heiligen Orte ihrer Religionen liegen außerhalb Indiens." (Schiessl 2013: 16) 


\subsection{Schaffung einer Atmosphäre des Misstrauens und Argwohns}

Hindutva als politische Größe ist relativ neu, bis in die 1970er Jahre bestand es nur als religiöses Konzept. Die 1980 gegründete BJP, die den politischen Anspruch vertritt, dass Indien nur den Hindus gehöre und mit politischen Mitteln versucht, „die nationalistischen und fundamentalistischen Ideen des RSS umzusetzen“"(Ceming 2004: 111), wuchs in den 1990er Jahren zur stärksten politischen Kraft Indiens heran. 2005 bis 2014 noch durch die Kongresspartei gebremst, ist die BJP nach dem Wahlsieg Modis wieder dabei, die Bundespolitik Indiens zu bestimmen. Die Hindutva-Agenda, deren Ziel die Abschaffung des „multiplen Nationsbegriffes“ (Six 2001: 73) nach der Vorstellung Nehrus und die Schaffung einer Nation der Hindus (Hindu Rashtra) ist, erhält dabei durch die BJP neue Impulse. Um die Mehrheit der Hindu-Stimmen für sich zu gewinnen, wird die Wählerschaft entlang religiöser Linien gespalten. Das heißt, Hinduismus versus Islam, Islam versus Christentum, Sikhismus versus Jainismus, aber auch zwischen Zugehörigen verschiedener Kasten (vgl. Schiessl 2013: 8). So lassen sich Wählerstimmen hinter Parteien vereinigen, die über Kasten und Landesgrenzen hinausgehen. Diese Polarisierung interreligiöser Grenzen zum Zwecke der politischen Interessenvertretung wird Kommunalismus genannt. ${ }^{10}$ „Kommunalismus ist ein Begriff, der hauptsächlich für Phänomene in Süd- und Südostasien verwendet wird. [...] Er bezeichnet eine Ideologie, die aus einer religiösen, kulturellen oder sozialen Gemeinschaft eine ökonomische und politische Ganzheit konstruiert, die sich maßgeblich über die Abgrenzung zum Anderen bestimmt“ (Schiessl 2013: 8).

Kommunalismus ist heute leider ein fester Bestandteil in Indiens politischer Landschaft (vgl. Seema 2014), dessen traurige Höhepunkte sich wie ein roter Faden durch die jüngste Geschichte ziehen: angefangen mit der wechselseitigen Ermordung hunderttausender MuslimInnen und Hindus nach der Teilung Indien-Pakistans (1949), gefolgt von blutigen Auseinandersetzungen im Bundesstaat Assam gegen muslimische MigrantInnen aus Bangladesch (1980er), der Jagd auf tausende Sikhs nach der Ermordung Indira Gandhis (1984), den Gewaltexzessen nach der Zerstörung der Moschee in Ayodhya ${ }^{11}$ (1992), dem

10 Kommunalismus unterscheidet sich von Faschismus insofern, als Hindunationalisten, deren Ideologie zu Kommunalismus führt, sich niemals offen zu Rassismus bekennen würden. Im Gegenteil behaupten Hindunationalisten, mit ihrer Ideologie der nationalen Hindu-Einheit Kommunalismus gerade zu bekämpfen. Zur expliziten Auseinandersetzung mit Hindunationalismus und Faschismus siehe Framke/Tschurnev (2010: 71ff.).

11 Ayodhya gilt in der Mythologie als Geburtsort des Gottes Rama, der dort vor gut 900.000 Jahren das Licht der Welt erblickt haben soll. 
Pogrom an MuslimInnen in Gujarat $(2002)^{12}$ oder der Verfolgung zehntausender ChristInnen im Bundesstaat Orissa (2008).

Mit jedem kommunalistischen Gewaltakt ist die BJP stärker geworden (vgl. Ceming 2004: 110), bis man ab 1996 bzw. 1998 mit der Wahl Vajpayees zum ersten BJP-Premierminister von einer Volkspartei sprechen kann. Daran wird deutlich, dass interreligiöse Konflikte nicht zufällig entstehen, sondern als Instrument der politischen Massenmobilisierung eingesetzt werden. „Lügen und Verzerrungen der Wahrheit zielen darauf ab, Misstrauen zu wecken und Hass zu schüren, und so eine explosive Atmosphäre zu schaffen“" (Seema 2014), die sich im Wahllokal auszahlt.

\subsection{Vom richtigen Handeln oder: Was bisher geschah}

Die Grundhaltung der HindunationalistInnen und ihr Geschichtsverständnis ist ein defensives. Die Ressentiments gegenüber Modernisierungsprozessen ${ }^{13}$ und dem sich wandelnden Wertekanon sowie der Hass gegenüber dem Anderen (den MuslimInnen), resultieren aus einem Bündel an Gründen. Zum einen existiert bei vielen Hindus ein nicht zu unterschätzender Respekt vor dem Zusammenhalt innerhalb der muslimischen Community, bei gleichzeitiger Empörung über die jahrhundertelange Vorherrschaft des Islams auf dem indischen Subkontinent (etwa 1206 bis 1858), verbunden mit der Trauer über die Teilung von Indien und Pakistan. Zum anderen gibt es eine dem Kastensystem zugrundeliegende Angst vor Verunreinigung, das heißt vor einer kulturellen Überfremdung, die teilweise als Verschwörung wahrgenommen wird (vgl. Kakar 2011: 134). Hinzu kommt ein intensives Gefühl der Benachteiligung im eigenen Land. Alle Quotierungen und Minderheitenprogramme werden dementsprechend als Diskriminierung der Hindu-Bevölkerung identifiziert. ${ }^{14}$ Außerdem geben RSS-HistorikerInnen eine stark verkürzte Sicht der indischen Besiedlungsgeschichte aus, welche wiederum die Hindus als Opfer erscheinen lässt. So sei die religiöse Vielfalt in Indien nicht durch Immigration wie in den USA entstanden, sondern durch Konversion. Die Mogul-Herrschaft hätte mit dem Schwert konvertiert, das Christentum

12 Für viele ist die Mitverantwortung Narendra Modis als damaliger Chief-Minister bis heute ungeklärt.

13 Modi hingegen verkörpert den in wirtschaftlichen Fragen aufgeschlossenen Parteiflügel. „Einmal im Besitz des Sessels des Premierministers lernte die BJP rasch in opportunistischer Manier, auf die politisch äußerst einflussreiche, vorwiegend städtische Mittelschicht Rücksicht zu nehmen und sich von den einstigen Feldzügen gegen das internationale Kapital zu verabschieden." (Six 2001: 110)

14 Nicht-Hindus in Indien verdienen keine Privilegien, keine Vorzugsbehandlung und noch nicht einmal Bürgerrechte, schrieb Golwalkar schon 1938. 
mit finanziellen Anreizen (vgl. The Economist 2015). Dies müsse rückgängig gemacht und gegenwärtige Konversionspraktiken unterbunden werden. Nach dem Wahlsieg Modis fühlt der RSS nun Rückenwind und schafft es, durch informelle Kanäle - sowohl Modi als auch die meisten BundesministerInnen waren lange Jahre im RSS aktiv - Parlamentsdebatten und Gesetzesentwürfe zu beeinflussen (vgl. Hein 2014). Nationale Schlachtverbote für Rinder sowie Antikonversionsgesetze, welche in einigen Bundesländern bereits existieren, stehen neben der Abschaffung des Sonderstatus für Jammu und Kaschmir ganz weit oben auf dem hindunationalen Wunschzettel. Yogi Adityanath, ein radikaler BJP-Parlamentarier und Hindu-Priester, tut sich beim Konversionsthema besonders hervor: „Wir werden einhundert Muslime für jeden konvertierten Hindu konvertieren“ (ebd.). Laut indischen Zeitungen kommt es in letzter Zeit häufig zu hinduistischen Massenkonversionen, welche im Sinne der HindutvaIdeologie nicht als Missionierung, sondern als Heimkehr oder Re-Konversion (gharwapsi) verstanden werden. Andersgläubige bzw. „fehlgeleitete“ InderInnen müssten nur ihren inneren Hindu (wieder-)entdecken. Mit Anspielung auf den Rindfleischkonsum von MuslimInnen und ChristInnen heißt es dazu sogar, ein Hindu könne „mit der Verhinderung einer Konvertierung fünf Kühe retten.“ (Kakar 2011: 136)

Um der angeblich überproportionalen Fertilität muslimischer InderInnen etwas entgegenzusetzen, forderte ein anderer religiöser Führer, der BJP-Parlamentarier Sakshi Maharaj jüngst, dass jede Hindufrau mindestens vier Kinder haben solle (vgl. The Economist 2015). In diesem Zusammenhang ist auch immer wieder vom Love Jihad die Rede. Das heißt „Beziehungen oder Liebesgeschichten zwischen Muslimen und Hindus werden als Strategie zur Ausweitung des Islam interpretiert“ (Schiessl 2013:24). Dem treten die Hindu-Nationalisten mit ähnlichen Ideen entgegen: „Die hinduistische Jugendorganisation Bajrang Dal fordert Hindu-Männer auf, gezielt Christinnen und Musliminnen zu heiraten, damit diese konvertieren" (Radunksi 2015).

Ähnlich wie der Mörder Mahatma Gandhis, ein RSS-Mitglied, der sich über die Entspannungspolitik gegenüber Pakistan ereiferte, schrecken auch heute die organisierten Hindunationalisten vor Gewalteinsatz gegenüber allen, die der Idee vom Hindu-Staat im Wege stehen, nicht zurück. Es geht um Einschüchterung sowie markige Präsenz - allein in Delhi kam es seit dem Wahlsieg der BJP zu mehreren Anschlägen auf Kirchen (vgl. Radunski 2015) -, aber auch um gezielte Beseitigung von Gegnern, wie die Morde an Govind Pansare, Narendra Dabholkar und Malleshappa Kalburgi belegen. Hiermit sei nicht gesagt, dass die Ermordung dieser linken und Aufklärungs-AktivistInnen allesamt auf das Konto radikalhinduistischer Gruppierungen geht. Die polizeilichen Ermittlungen dauern noch an; politische Motive sind allerdings naheliegend. Die eigene Gewalt 
wird von Hindunationalisten dabei immer als notwendige Reaktion empfunden, womit alle Verantwortung auf die Seite der anderen geschoben wird. Denn dem Hindu-Fundamentalisten geht es weniger um die richtige Theorie als um die richtige Praxis: „Der Hindufundamentalismus ist ein Fundamentalismus der Orthopraxie und nicht der Orthodoxie" (Ceming 2004: 113). ${ }^{15}$

Aufgrund der erwähnten beispielhaften Vorkommnisse ist in der indischen Öffentlichkeit in jüngster Zeit ein Klima der Verunsicherung entstanden, das medial darin gipfelte, dass eine ganze Reihe namhafter InderInnen ihre nationalen Preise und Auszeichnungen zurückgaben, verbunden mit der Forderung nach dem Ende kommunalistischer Gewalt: Den Anfang machten die SchriftstellerInnen, dann folgten die ProfessorInnen, WissenschaftlerInnen, RegisseurInnen und SchauspielerInnen.

Geht man davon aus, dass Modi und vermutlich auch ein Großteil der BJP-Elite sich inzwischen als gemäßigt verstehen, stellt sich die Frage, warum der Premierminister jeweils Wochen, teilweise sogar Monate braucht, um kommunalistische Ereignisse öffentlich zu verurteilen. Die Antwort ist gleichermaßen trivial wie problematisch: Weil er dem RSS und den BJP-HardlinerInnen etwas schuldet. Zwischen dem RSS und der BJP besteht ein Eltern-Kind-Verhältnis und die Zusammenarbeit läuft auf zwei Ebenen: Ideologie und Personal (vgl. Varghese 2014). Ohne den RSS wäre Modi niemals Premierminister geworden. Zwar hat er öffentlich verkündet, sich auf das ökonomische Programm zu konzentrieren, aber es gibt ein religiöses Establishment, auf dessen Unterstützung er nach wie vor angewiesen ist. Modi muss auf die Forderungen seiner Stamm-Wählerschaft Rücksicht nehmen, die das eigene rechte Kulturprojekt (Hindutva) möglichst schnell durchsetzen wollen und dazu auf die Mobilisierung durch kommunalistische Gewalt setzt. Indem die Regierung auf dem rechten, also orangefarbenen Auge blind ist, fühlen sich fundamentalistische Akteure sicher. Der mangelnde Wille zum klaren Wort, oder sogar zu Gegenmaßnahmen, deckt die Geschehnisse der Straßengruppen nicht nur, sondern motiviert sie zum Weitermachen. Selten werden Straftäter ermittelt oder verurteilt. Oftmals scheint es vielmehr eine Übereinkunft zwischen Polizei, lokaler Regierung und hindunationalistischen Gruppen zu geben.

Ein weiteres Indiz für den wirklichen Machtwechsel sind die Einflussnahmen im Bereich der Kultur-, Sozial- und Bildungspolitik, welche den Aufbau einer rechten Hegemonie seitens der BJP-Regierung nahe legen. Neben den oben erwähnten, mehr symbolischen Themen des Rindfleisch- und Konversionsverbots

15 Der Fokus auf das richtige Handeln (Orthopraxie), statt auf den richtigen Glauben (Orthodoxie) hat auch damit zu tun, dass es in Indien keine wirkliche Trennung zwischen dem religiösen und dem säkularen Leben gibt (vgl. Tharoor 2005: 362). 
scheint die Neubesetzung relevanter Institutionen im Sinne einer „Safranisierung", also die Etablierung der eigenen hinduistischen Leitkultur, das eigentliche Anliegen des RSS zu sein (vgl. Puniyani 2015). So wurden Schulbücher neu formuliert, um eine andere Geschichtsschreibung einzuleiten. Aus dem Taj Mahal wird ein Shiva Tempel namens Tejo Mahalay, das Alphabet wird nach hinduistischen Götternamen gelernt, allen Pflanzen wird eine Seele zugeschrieben und Passagen, die das Kastensystem kritisch beleuchten, werden gestrichen (vgl. Schiessl 2013: 25f.). Der Sanskritunterricht wurde wieder eingeführt, teilweise auf Kosten der deutschen Fremdsprache, und wichtige Schlüsselposten, zum Beispiel im nationalen Institut für Geschichte, Bildung und Forschung oder im Institut für Film und Fernsehen, wurden mit Personen besetzt, die zwar wenig qualifiziert, dafür aber ideologisch auf Linie sind. ${ }^{16}$ Theorien setzen sich eben nicht durch, weil sie wahr oder falsch sind, sondern wenn Macht dahinter steht.

\section{Hindu-Ideologie und/oder Big Business?}

Innerhalb der hindunationalistischen Bewegung gibt es verschiedene Strömungen, deren Hauptlinien zwischen ökonomischem Liberalismus und kultureller Reaktion bzw. der Neuerfindung traditioneller Werte verlaufen (vgl. Framke/ Tschurenev 2010: 73). Obwohl beide Pole für die Macht im Zentrum aufeinander angewiesen sind, gibt es Spannungen, denn das internationale Kapital schätzt nationalistische Protektionen nicht; andererseits entspricht die konsumorientierte, liberale und säkulare Mittelklasse Indiens, insbesondere mit ihrer Offenheit gegenüber der westlichen Sexualmoral, kaum mehr den Vorstellungen der Hindu-NationalistInnen (vgl. Kakar 2011: 147f.). Es stellt sich also die Frage, ob die Hindutva-Ideologie den Imperativen des globalen Finanzkapitals im Wege steht. Wie groß ist deshalb der Druck auf Modi? Schließlich muss er den Unternehmer-Freund spielen, und zugleich als nationaler Beschützer und Identitätsstifter funktionieren. Daran zeigt sich noch einmal das hypermedialisierte Bild des Premiers, auf dessen Oberfläche permanent die ideologischen Risse sichtbar werden. Umgekehrt wirkt seine Medienfigur auch als Deckmantel, unter dem sich problematische Reformen kaschieren lassen.

16 „Die intellektuelle Führung des RSS ist so weit von aktuellen Diskursen der Moderne entfernt, dass sie beinahe komisch wirkt" (Shoaib 2014; Übers.: A.W.). Die Forderung nach mehr Hindu-Kindern, wo es eigentlich um eine Eindämmung des Bevölkerungswachstums gehen müsste, mag dafür ein Beispiel sein. Zum Defizit konservativer Intellektueller siehe auch Sampath 2015. 
Nicht nur im Falle Indiens zeigt sich, dass eine kapitalistische Wirtschaftspolitik sehr gut mit einer konservativen Agenda hinsichtlich Kultur und Religion zusammengeht. Die Parallelen zu Indien liegen insbesondere bei der Türkei auf der Hand. Sowohl Modi als auch der türkische Präsident Recep Tayyip Erdoğan traten ihre Regierung mit dem Versprechen an, die Korruption und Misswirtschaft der säkularen Vorgängerregierungen zu beenden, beide taten sich (zunächst) durch eine Entspannungspolitik gegenüber ihren regionalen Nachbarn hervor und verpacken ihre Ideologie des Wachstums und Konsumismus mit religiösen Gesten und Symbolen. Sowohl AKP als auch BJP dienen mit ihrer zentralisierten, autoritären Organisation als Agent des flexiblen Kapitals (vgl. Ghosh 2014). So lässt sich in Indien aktuell die Entwicklung eines HindutvaKapitalismus beobachten, welcher Hindutva 2.0 (Varghese 2014) oder MarktHinduismus genannt werden kann.

Bei der Integration der neoliberalen Ideologie in die Partei war Modi mit seinem Gujarat-Beispiel ein entscheidender Vorreiter. Unternehmen wollen über billige Arbeit und billiges Land ohne Umweltauflagen verfügen, Effizienz bringende Investitionen in Bildung und Infrastruktur tätigen, keine Steuern zahlen und keine Rücksicht auf Arbeitnehmerrechte nehmen müssen. In Gujarat wurden diese Kriterien unter Modis Ministerpräsidentschaft Realität, was einige UnternehmerInnen zu MilliardärInnen machte, während zahlreiche Bäuerinnen und Bauern sowie FischerInnen gegen den Verlust ihres Weidelands und die Verschmutzung des Meeres kämpften. „Die Hindunationalisten bilden mehr als offensichtlich eine florierende Allianz mit der neoliberalen Wirtschaft auf Kosten von Arbeiter- und Minderheitsrechten." (Schiessl 2013: 21). Nach dem Wahlsieg auf Bundesebene befand sich die indische Börse konsequenterweise im Freudentaumel. Von Goldman Sachs bis Citibank korrigierten alle großen Investmenthäuser ihre Prognosen für die indische Wirtschaft nach oben. ${ }^{17}$ Unter Manmohan Singh stieg die Wachstumsrate bis auf zehn Prozentpunkte im Jahr 2010, fiel aber in den vergangenen drei Jahren wieder auf fünf Prozentpunkte ab. Für die Corporate World war der entscheidungsschwache Singh also eine Hürde, die beseitigt werden musste. „Der Widerstand musste gebrochen und beseitigt werden. Das Kapital benötigt einen Mann, der die letzte Meile gehen kann. Deshalb hat die Großindustrie Millionen in Modis Wahlkampagne gesteckt“ (Roy 2014; Übers.: A.W.).

17 Ähnlich verhält es sich auch mit der Weltpolitik. Galt Modi bis 2013 in der EU und den USA aufgrund seines Macho-Hindunationalismus noch als persona non grata, scheint er jetzt als Wachstumsgarant ein gern gesehener Gast zu sein, sodass Modi es innerhalb seines ersten Amtsjahres bereits auf 19 Staatsbesuche brachte. 
Doch „private Super-Autobahnen und Schnellstraßen zu bauen ist nicht das Gleiche, wie die notwendige Infrastruktur für die Bevölkerung bereitzustellen“ (Shiva 2014: 56). Wie schafft es die Regierung, ihr Wahlversprechen vom großen Wandel umzusetzen? Im Sinne der Hindutva-Ideologie beschwört Modi dazu die nationale Einheit. „Somit wird ein systeminhärenter Konflikt negiert und jegliche Verantwortung von den Schultern der aufstrebenden Eliten genommen " (Schiessl 2013: 20). Gesellschaft wird nicht vertikal differenziert, zwischen oben und unten, sondern horizontal: wir und die anderen. Kommt es zu Interessenkonflikten, werden diese kommunalistisch interpretiert: „Ihr bekommt keine Studien- oder Arbeitsplätze, weil Muslime oder Angehörige der 'Scheduled Castes' sie euch durch die Quotenregelung wegnehmen." (Schiessl 2013: 21) Auch sonst passt die Hindutva-Ideologie sehr gut zum neoliberalen Staatsverständnis: Bestehende wirtschaftliche und soziale Verhältnisse werden nicht infrage gestellt. Weder das koloniale Erbe in Sachen Grundbesitz noch das hierarchische Kastensystem sind für die BJP ein Thema. „Der Aufstieg der unteren Kasten in gehobene Positionen verstößt nach Meinung der BJP gegen die heilige Ordnung, da die Aufgabe der niederen Kasten im Dienen und nicht im Herrschen besteht, so wie es eben im Gesetz des Manu festgeschrieben ist." (Ceming 2004: 111) Hindutva 2.0 ist also ein Wirtschaftsmodell, welches Wachstum und Wohlstand mit einem HinduPrimat kombiniert und die passende Geschichte dazu erfindet. Konkret bedeutet dies, dass der neue nationale Kleinwagen (Tata Nano), der inzwischen in Gujarat produziert wird, mit dem Gott Krishna verglichen wird, welcher ebenfalls in Dwarka, Gujarat, aufgewachsen sein soll (vgl. Varghese 2014).

Wenn es gut läuft und Modi zwei Legislaturperioden mit stabiler Mehrheit und ohne religiöse Kernschmelze schafft, hat Indien danach ein paar Straßen, Toiletten, Flughäfen mehr und eine stabile Stromversorgung sowie eine Müllabfuhr. Wenn es so läuft wie bisher, versickert selbst das dafür aufgebrachte Geld in der Korruption von Bundesstaaten und Großindustriellen, und alles bleibt, wie es ist. Wenn es schlecht läuft, steht Indien eine Phase autoritärer Politik bevor. „Modis Plan A ist der Erfolg an der ökonomischen Front, und falls das nicht funktioniert, wird der Fokus auf Hindutva vermutlich zu einem wichtigen Plan B“ (Vij 2014; Übers.: A.W.). In jedem Fall bleibt der Umgang mit Minderheiten und bürgerlichen Freiheitsrechten der wichtigste Indikator für die Bewertung von Indiens Politik. 


\section{Literatur}

Amin, Samir (2007): Weltmacht Indien? Der Subkontinent zwischen kolonialem Erbe und globalem Aufstieg. In: Blätter für deutsche und internationale Politik 51(6): 705-716.

Barry, Ellen (2015): After a year of outsize expectations. Narendra Modi adjusts his plan for India. In: New York Times. URL: http://www.nytimes.com/2015/05/26/world/asia/after-a-year-ofoutsize-expectations-modi-adjusts-his-political-course-for-india.html, Zugriff: 13.09.2015.

Ceming, Katharina (2004): Hinduismus. Auf dem Weg vom Universalismus zum Fundamentalismus? In: polylog. Zeitschrift für interkulturelles Philosophieren, Nr. 12: 99-114.

Framke, Maria/Tschurenev, Jana (2010): Umstrittene Geschichte. (Anti-)Faschismus und (Anti-) Kolonialismus in Indien. In: PROKLA 40(1): 67-83.

Ghosh, Amitav (2014): Parallel Journeys? Turkey's experience of AKP rule and its portents for India under the BJP. URL: http://amitavghosh.com/blog/?p=6857, Zugriff: 05.12.2015.

Gupta, Dipankar (2011): Mistaken Modernity. India between worlds, Noida.

Harneit-Sievers, Axel (2015): Wachstum, Ernüchterung und Verunsicherung: Ein Jahr Modi in Indien. URL: https://www.boell.de/de/2015/05/19/wachstum-ernuechterung-und-verunsicherung-ein-jahr-modi-regierung-indien, Zugriff: 1.12.2015.

Hein, Christoph (2014): Hindu-Nationalisten gehen in die Offensive. In: Frankfurter Allgemeine Zeitung. URL: http://www.faz.net/aktuell/politik/ausland/asien/nationalisten-wollen-reinenhindu-staat-in-indien-13341511-p2.html?printPagedArticle=true\#pageIndex_2, Zugriff: 25.11.2015.

Hörig, Rainer (2007): Hinduismus ist anders!, In: SympathieMagazin, Nr. 54: 4-5.

Kakar, Sudhir/Kakar, Katharina (2011): Die Inder. Portrait einer Gesellschaft, München.

Khilnani, Sunil (2014): Nehru, Year of Death, 2014?, In: Outlook 54(45): 16-18.

Prasannarajan, S. (2014): The Prime Mover of an India rediscovered and the challenges of being modern. In: Open 6(51): 10-17.

Puniyani, Ram (2015): India should say no to the RSS version of history. In: The Dawn. URL: http://www.dawn.com/news/1173921, Zugriff: 2.12.2015.

Purkayastha, Prabir (2015): A year of Modi: Impoverishes people, lost hopes (Gespräch mit Harsh Mandar). In: The Real News. URL: http://therealnews.com/t2/index.php?option=com_cont ent\&task=view\&id=31\&Itemid=74\& jumival=13967, Zugriff: 22.11.2015.

Radunski, Michael (2015): Da brannte die Kirche lichterloh. In: taz.die tageszeitung. URL: http:// www.taz.de/!5012948/, Zugriff: 5.12.2015.

Roy, Arundhati (2014): Now we have a democratically elected totalitarian government. In: The Dawn. URL: http://www.dawn.com/news/1108001, Zugriff: 5.10.2015.

Sampath, G. (2015): The missing conservative intellectuals. In: The Hindu. URL: http://www. thehindu.com/opinion/lead/the-missing-conservative-intellectuals/article7461274.ece, 1Zugriff: 23.11 .2015 .

Schiessl, Franziska (2013): Kommunalismus, Hindunationalismus und Widerstand. Auf den Spuren der Hindunationalisten und der Suche nach Widerstand, Rosa Luxemburg Stiftung South Asia working paper series, Berlin.

Seema, Mustafa (2014): Der Kampf um ein säkulares Indien. URL: https://www.boell.de/ de/2014/02/25/der-kampf-um-ein-sekulaeres-indien, Zugriff: 2.12.2015.

Shoaib, Daniyal (2014): Modi wants to be the new Nehru, but what is his plan for a new republic? URL: http://scroll.in/article/678076/modi-wants-to-be-the-new-nehru-but-what-is-his-planfor-a-new-republic, Zugriff: 2.12.2015.

Six, Clemens (2001): Hindu-Nationalismus und Globalisierung. Diezwei Gesichter Indiens: Symbole der Identität und des Anderen, Frankfurt/M.

Skischally, Bernd (2014): Sein Erfolg ist eine Illusion. In: WIRED Germany 1(1): 110-115. 
Thackerey, Bal: The Nation of Our Dreams. In: The Indian Express, 11.10.1998.

Tharoor, Shashi (2005): Eine kleine Geschichte Indiens, Frankfurt/M.

The Economist (2015): The Hindutva rate of growth. URL: http://www.economist.com/news/ asia/21639567-narendra-modi-finds-his-economic-ambitions-jeopardised-his-partys-ideologyhindutva-rate, Zugriff: 21.11.2015.

Tilman, Steffen (2015): Indiens twitternder Premier. In: Die Zeit. URL: http://www.zeit.de/ politik/ausland/2015-05/narendra-modi-indien-twitter-facebook, Zugriff: 10.11.2015.

Trojanow, Ilja (2007): Hinduismus ist anders! In: SympathieMagazin, Nr. 54: 3.

Varghese, K. George (2014): A Hindutva variant of neo-liberalism. In: The Hindu. URL: http:// www.thehindu.com/opinion/lead/a-hindutva-variant-of-neoliberalism/article5868196.ece, Zugriff: 15.11.2015.

Vij, Shivam (2014): Modi's plan A will be economy. If that does not work, Hindutva. In: scroll.in. URL: http://scroll.in/article/664475/modis-plan-a-will-be-economy-if-that-does-not-workhindutva, Zugriff: 23.11.2015.

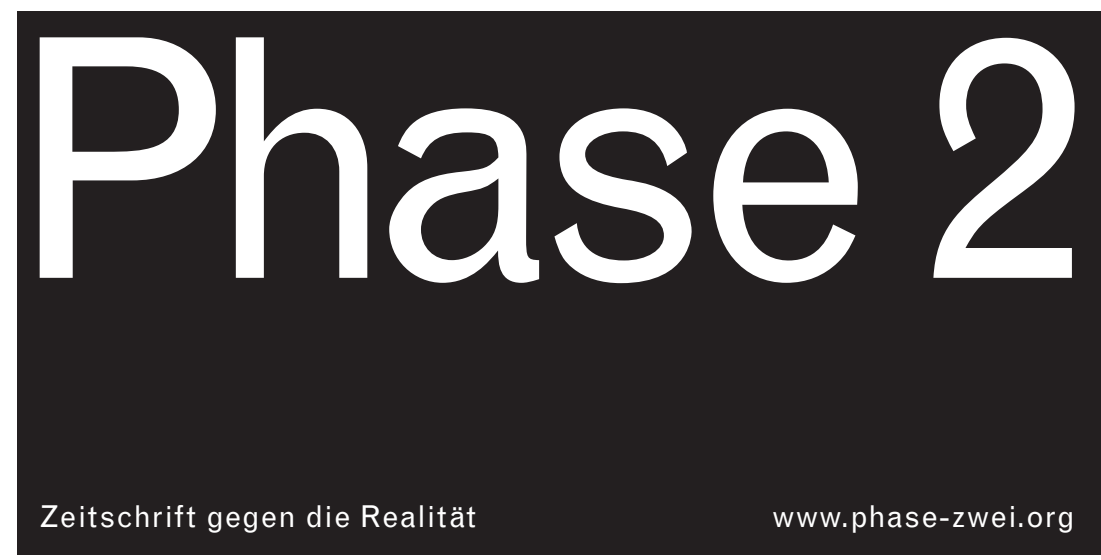

